\title{
Overexpression of Tiam1 predicts poor prognosis in patients with esophageal squamous cell carcinoma
}

\author{
HUAIMIN LIU ${ }^{1}$, GUIRONG SHI ${ }^{3}$, XIAOLI LIU ${ }^{4}$, HUI WU ${ }^{5}$, QINXIA FAN $^{1}$ and XIN WANG ${ }^{2}$ \\ Departments of ${ }^{1}$ Oncology and ${ }^{2}$ Radiotherapy, The First Affiliated Hospital of Zhengzhou University, Zhengzhou 450052; \\ ${ }^{3}$ Department of Nursing, Shangqiu Medical College, Shangqiu 476100; Departments of ${ }^{4}$ Oncology and \\ ${ }^{5}$ Radiotherapy, Henan Province Tumor Hospital, Zhengzhou 450008, P.R. China
}

Received September 29, 2010; Accepted November 15, 2010

DOI: $10.3892 /$ or.2010.1122

\begin{abstract}
Accumulating evidence has demonstrated that Tcell lymphoma invasion and metastasis 1 (Tiam1) plays an important role in the occurrence and development of several different tumors; however, to date, little research has been done to verify the potential role of Tiam1 as a prognostic marker for esophageal squamous cell carcinoma (ESCC). In the present study, we examined the expression of Tiam1 in ESCC tissues by immunohistochemistry, in situ hybridization, semi-quantitative RT-PCR and Western blotting methods and investigated the correlation between Tiam1 levels and prognosis of patients with ESCC. Tiam1 exhibited high expression in ESCC tissues, whereas the normal esophageal tissues showed negative or weak Tiam1 expression. Additionally, Tiam1 mRNA and protein expression levels were both significantly correlated with histology grade, clinical staging and lymph node metastasis (all $\mathrm{P}<0.05$ ), but not related to age and gender (both $\mathrm{P}>0.05$ ). Further, ESCC patients with strong Tiam1 mRNA $(\mathrm{P}=0.000)$ and protein $(\mathrm{P}=0.000)$ expression had a poorer prognosis than those with weak expression. These findings demonstrate that Tiam1 may be used as molecular marker for predicting the prognosis of patients with ESCC.
\end{abstract}

\section{Introduction}

Esophageal carcinoma, one of the most aggressive carcinomas of the gastrointestinal tract, is the sixth most common cause of cancer related death worldwide $(1,2)$. Esophageal squamous cell carcinoma (ESCC) as the main histological type of esophageal carcinoma occurs at a very high frequency in China, South Africa, France and Italy (3), and especially in

Correspondence to: Dr Xin Wang, Department of Radiotherapy, The First Affiliated Hospital of Zhengzhou University, 40 Daxue Road, Zhengzhou, Henan 450052, P.R. China

E-mail: wxgoal@126.com

Key words: T-cell lymphoma invasion and metastasis 1, esophageal squamous cell carcinoma, clinicopathological features, prognosis
Linzhou city of Henan Province, China (4). Although recently there is an increase in adenocarcinomas of esophagus in Western countries, most esophageal cancers are still squamous cell carcinomas in the world (5-7). Currently, although therapy strategies have improved, the prognosis of patients with ESCC is still poor and the 5-year survival rate is decreasing (7). There is, therefore, an urgently need for new molecular markers to help improve the prognosis of patients with ESCC.

It has been reported that T-cell lymphoma invasion and metastasis 1 (Tiam1) participates in morphological transformation, cell motility and invasive behavior in many cell types, such as T-lymphoma cells, fibroblasts and epithelial cells (8-11). Increasing evidence has focused on the regulation of Tiam1 expression, as well as the role of Tiam 1 in cancer progression and metastasis that Tiam 1 specifically activates Rho-like GTPases (e.g., Rac1) and Tiam1-Rac1 pathway affects the migration and invasion of many tumors, such as nasopharyngeal carcinoma (12), breast cancer (13), retinoblastoma (14) and hepatocellular carcinoma (15). Additionally, overexpression of Tiam1 in tumor cells, implying a poor prognosis, has been linked to multiple different tumors (12-15). However, to date, few studies have been performed to investigate whether Tiam1 plays a major role in the occurrence and development of ESCC and whether its overexpression implies poor prognosis of the patients with ESCC. The purpose of the present study was to determine the expression level of Tiam1 in ESCC tissues and to analyze the prognostic value of Tiam1 in the patients with ESCC.

\section{Materials and methods}

Patients and tissue samples. A total of 173 cases of paraffinembedded ESCC tissues, 95 cases of para-carcinoma tissues and 173 cases of normal esophageal tissues utilized in this study were obtained from The First Affiliated Hospital of Zhengzhou University from 2005 to 2009, which were histopathologically and clinically confirmed as ESCC by The First Affiliated Hospital of Zhengzhou University. The patients did not receive any form of treatment prior to surgery, and the clinical data about the samples are listed in Table I.

Immunohistochemistry (IHC) staining of Tiaml gene. Expression of the Tiam1 gene was investigated by streptavidin- 
Table I. Clinicopathological characteristics of patients with ESCC.

\begin{tabular}{lc}
\hline Features & No. of patients $(\mathrm{n}=173)(\%)$ \\
\hline Gender & $95(54.9)$ \\
Male & $78(45.1)$ \\
Female & \\
Age (years) & $129(74.6)$ \\
$\geq 60$ & $44(25.4)$ \\
$<6$ & \\
Histological grade of tumor & $51(29.5)$ \\
Well-differentiated & $47(27.2)$ \\
Moderately-differentiated & $75(43.4)$ \\
Poorly-differentiated & \\
Tumor stage & $70(40.5)$ \\
I and II & $103(59.5)$ \\
III and IV & \\
Lymph node metastasis & $97(56.1)$ \\
Yes & $76(43.9)$ \\
No &
\end{tabular}

biotin-peroxidase complex method according to a previous publication (16). Briefly, ESCC tissue sections were deparaffinized and non-specific bindings were blocked with $10 \%$ normal rabbit serum for $10 \mathrm{~min}$. The tissue sections were then incubated at $4^{\circ} \mathrm{C}$ overnight with anti-Tiam1 polyclonal antibody (Santa Cruz Biotechnology, Santa Cruz, CA) at a dilution of 1:50, and subsequently incubated with biotinylated goat anti-rabbit immunoglobulin at a concentration of 1:100 for $30 \mathrm{~min}$ at $37^{\circ} \mathrm{C}$. The slides were observed under the microscope and pictures were taken for analysis. Negative controls were performed by replacing the primary antibody by normal serum.

In situ hybridization (ISH). ISH was carried out according to previous descriptions $(16,17)$. In brief, the probe used for in situ hybridization comprised of a 180-bp region of Tiam1 cDNA (GenBank accession no.: NM_003253) amplified with specific primers (forward: 5'-AATCGTGCTGCTGAC GCCCT-3' and reverse: 5'-GGTGAGAAAGGCGAGGGG GC-3') and labeled with digoxigenin (Promega Corp., Madison, WI). The slides were deparaffinized, hydrated and then treated with $0.2 \mathrm{~mol} / 1 \mathrm{HCl}$ for $10 \mathrm{~min}$ at room temperature (RT), followed by digestion with proteinase K (100 mg/l) at $37^{\circ} \mathrm{C}$ for $15 \mathrm{~min}$. The slides were placed in hybridization solution containing $50 \%$ formamide, $4 \mathrm{X} \mathrm{SSC}, 5 \%$ dextran sulfate, 5X Denhardt's solutions, $200 \mathrm{mg} / \mathrm{ml}$ denatured salmon sperm DNA and Tiam1 probes (25-50 ng) and the hybridization reaction was completed overnight in a wet chamber at $42^{\circ} \mathrm{C}$. After hybridization, the slides were washed with 2X SSC and 1X SSC for $30 \mathrm{~min}$ each. Anti-digoxigenin antibody conjugated with alkaline phosphatase was added to the samples for $30 \mathrm{~min}$ at $37^{\circ} \mathrm{C}$. The purple-blue ISH signals were developed by incubating slides in NBT/BCIP for $15 \mathrm{~min}$. The slides were observed under the microscope and pictures were taken for analysis. The negative control was incubated with hybridization solution without Tiam1 probe.

Immunostaining score. All of the fields in the selected block were taken into consideration for assessment of immunostaining. The percentage of tumor cells stained of the total tumor cells was reported. Representative areas of each section were selected, and cells were counted in at least four fields at 400-fold magnification. Scoring based on the percentage of positive cells of Tiam1 staining was identified as $0,<5 \% ; 1,5-25 \% ; 2,25-50 \% ; 3,50-75 \%$; and $4,>75 \%$. The intensities of Tiam1 staining were scored as follows: $1+$, weak; $2+$, moderate; and $3+$, intense. The scores indicating percentage of positive tumor cells and staining intensity were multiplied to produce a weighted score for each case. Cases with weighted scores $<1$ were defined as negative, and the other cases as positive.

Semi-quantitative $R T-P C R$. Total RNA was isolated from ESCC tissues and normal esophageal tissues using TRIzol reagent (Invitrogen, Carlsbad, CA) following the manufacturer's instruction. Reverse transcription of total RNA $(10 \mu \mathrm{g})$ was performed for each sample using a First-Strand cDNA Synthesis Kit (Biotech Co., Shanghai, P.R. China), and cDNA was subjected to PCR for 30 cycles of amplification described as follows: $30 \mathrm{sec}$ at $94^{\circ} \mathrm{C}, 30 \mathrm{sec}$ at $56^{\circ} \mathrm{C}$ and $30 \mathrm{sec}$ at $72^{\circ} \mathrm{C}$. The PCR primers used for amplification of Tiam1 gene were 5'-CAGGACTCCACGGGGCCTCA-3' for forward primer and 5'-AGTGCTCCTCGCTCTCCGCA-3' for reverse primer (product size is $580 \mathrm{bp}$ ), and internal control $B$-actin primers were 5'-CGGGGTCACCCACACTG TGC-3' for forward primer and 5'-GGGCAGCGGAACCGC TCATT-3' for reverse primer (product size is $303 \mathrm{bp}$ ). The amplification products were separated on $1.5 \%$ agarose gels and visualized by ethidiumbromide staining, and semiquantitative analysis of band intensity was done with Gene Tools software (UVP, Inc., Upland, CA).

Western blotting. Western blotting was performed according to standard methods as described previously $(18,19)$. Briefly, ESCC tissues and normal esophageal tissues were lysed in a sample buffer (50 mM Tris-HCl, pH 8.0; $150 \mathrm{mM} \mathrm{NaCl}, 1 \%$ Triton X-100; $100 \mu \mathrm{g} / \mathrm{ml}$ PMSF) on ice for $30 \mathrm{~min}$. Lysates were then centrifuged for $5 \mathrm{~min}\left(12000 \mathrm{x} \mathrm{g}, 4^{\circ} \mathrm{C}\right)$. Protein concentration was determined by the Bradford method. An equal amount of tissues lysates (60 $\mu \mathrm{g} / \mathrm{lane})$ was electrophoresed through a $10 \%$ SDS-PAGE gel, and then transferred to nitrocellulose membranes (Amersham, Uppsala, Sweden). The membranes were then blocked in 5\% skimmed milk in PBS-T containing $0.05 \%$ Tween-20 at RT for $2 \mathrm{~h}$, and then incubated at RT for $2 \mathrm{~h}$ with corresponding primary antibodies including anti-Tiam 1 and anti- $\beta$-actin (all from Santa Cruz Co.) diluted in $1 \%$ skimmed milk in PBS-T, respectively, followed by incubation with horseradish peroxidase-linked goat anti-mouse $\mathrm{IgG}$, and the bands of specific proteins on the membranes were developed with DAB. Quantification of band intensity was performed using Gene Tools (UVP, Inc.). 


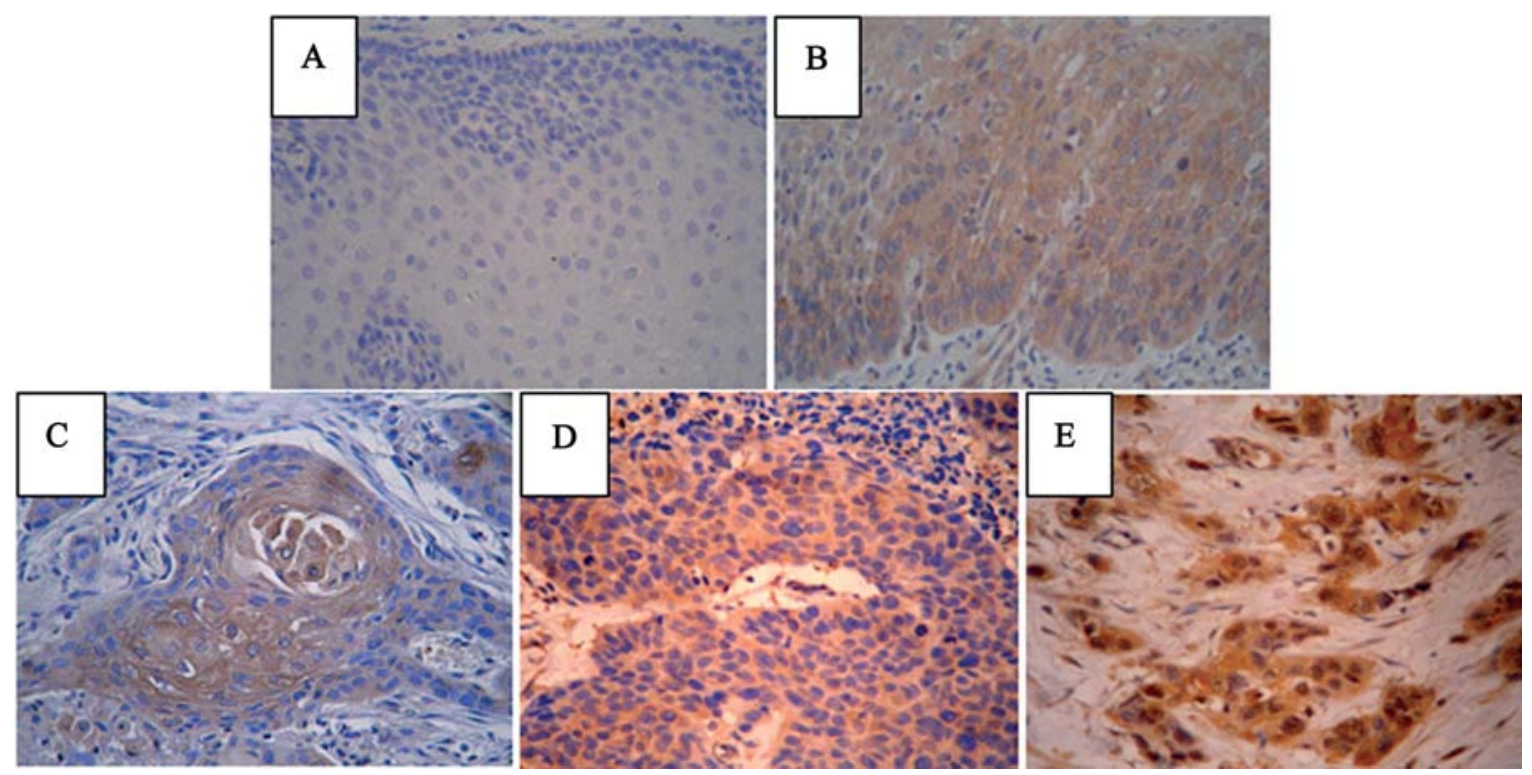

Figure 1. Immunohistochemical staining of Tiam1 protein in ESCC, para-carcinoma and normal esophageal tissues. (A) No Tiam1 protein staining was present in the normal esophageal tissues. (B) Moderate Tiam1 protein expression in the para-carcinoma tissues. (C) Strong positive Tiam1 immunostaining in welldifferentiated ESCC tissues. (D) Stronger positive Tiam1 immunostaining in moderately-differentiated ESCC tissues. (E) The strongest positive Tiam1 immunostaining in poorly-differentiated ESCC tissues.

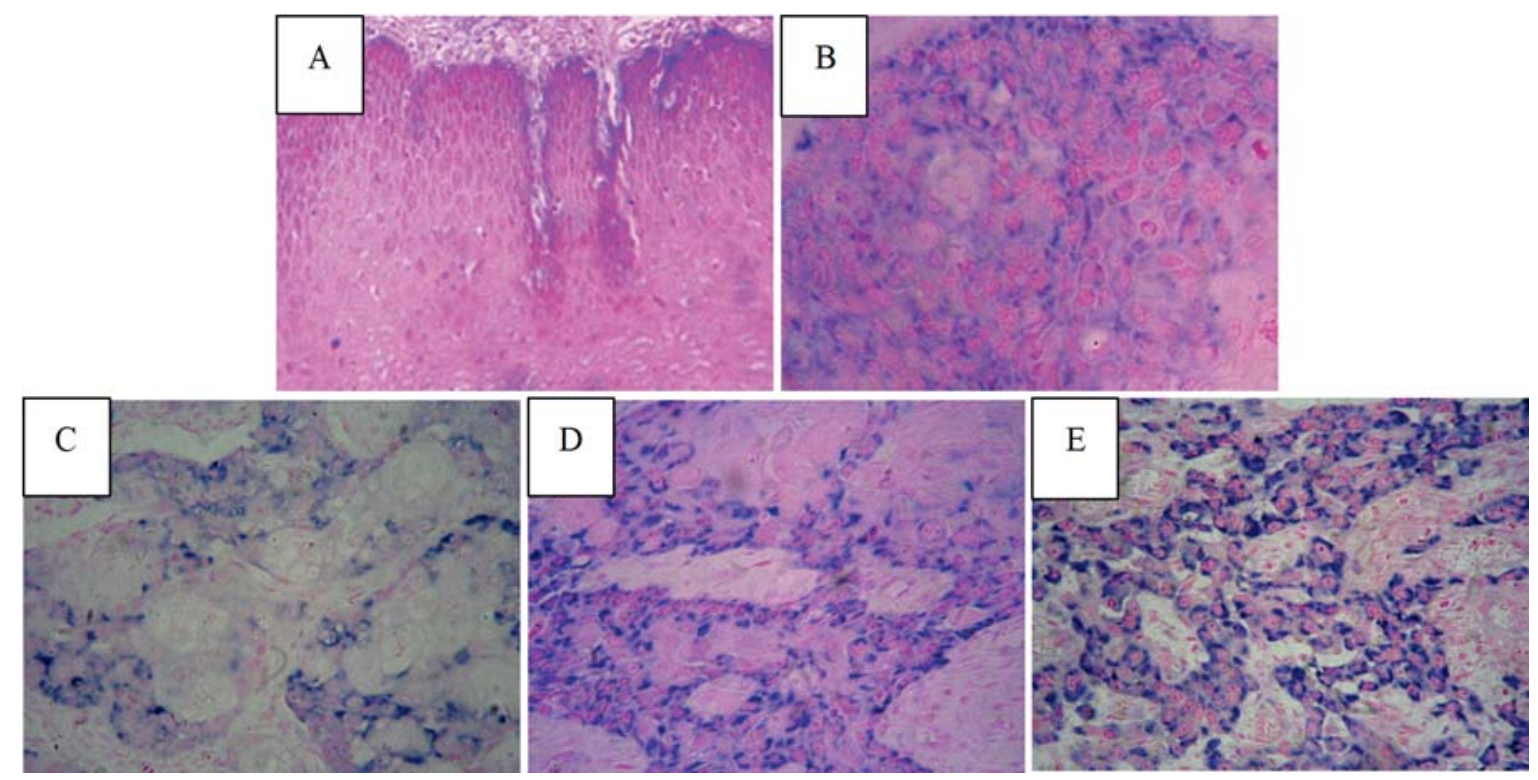

Figure 2. In situ hybridization staining of Tiam1 mRNA in ESCC, para-carcinoma and normal esophageal tissues. (A) No or weak Tiam1 mRNA staining was present in the normal esophageal tissues. (B) Moderate Tiam1 mRNA staining in the para-carcinoma tissues. (C) Strong positive Tiam1 mRNA staining in well-differentiated ESCC tissues. (D) Stronger positive Tiam1 mRNA staining in moderately-differentiated ESCC tissues. (E) The strongest positive Tiam1 mRNA staining in poorly-differentiated ESCC tissues.

Statistical analysis. The correlations between expressions of Tiam1 mRNA and protein with various clinicopathological parameters were evaluated with the $\chi^{2}$ test. Survival analyses were performed according to the Kaplan-Meier method. Cox proportional-hazard analysis was used for univariate and multivariate analysis to explore the effect of variables on survival. The SPSS 13.0 software was used for all statistical analyses and a P-value of $<0.05$ was considered significant.

\section{Results}

Clinicopathological features of ESCC. Clinicopathological features of ESCC and demographic characteristics of the patients from whom they were obtained are listed in Table I. Particularly, cases of male and female in this study were 95 $(54.9 \%)$ and $78(45.1 \%)$, respectively, 44 cases $(25.4 \%)$ were aged $<60$ years, whereas 129 were $\geq 60$ years old. Additionally, regarding histological grade of ESCC, well, 
Table II. Correlation between Tiam1 expression and the clinicopathological characteristics of the patients with ESCC.

\begin{tabular}{|c|c|c|c|c|c|}
\hline Characteristic & Total $(n=173)$ & $\begin{array}{l}\text { Tiam1 protein } \\
\text { expression }(\%)\end{array}$ & P-value & $\begin{array}{l}\text { Tiam1 mRNA } \\
\text { expression }(\%)\end{array}$ & P-value \\
\hline \multicolumn{6}{|l|}{ Gender } \\
\hline Male & 95 & $86(90.5)$ & & $80(84.2)$ & \\
\hline Female & 78 & $73(93.6)$ & 0.462 & $71(91.0)$ & 0.181 \\
\hline \multicolumn{6}{|l|}{ Age } \\
\hline$\geq 60$ & 129 & $119(92.2)$ & & $112(86.8)$ & \\
\hline$<60$ & 44 & $40(90.9)$ & 0.779 & 39 (88.6) & 0.755 \\
\hline \multicolumn{6}{|l|}{ Histologic grade } \\
\hline Well-differentiated & 51 & $40(78.4)$ & & $33(64.7)$ & \\
\hline Moderately-differentiated & 47 & $44(93.6)$ & & $43(91.5)$ & \\
\hline Poorly-differentiated & 75 & $75(100)$ & 0.000 & $75(95.6)$ & 0.000 \\
\hline \multicolumn{6}{|l|}{ Clinical stage } \\
\hline I and II & 70 & $60(85.7)$ & & $51(72.9)$ & \\
\hline III and IV & 103 & $99(96.1)$ & 0.014 & $100(97.1)$ & 0.000 \\
\hline \multicolumn{6}{|l|}{ Lymph node metastases } \\
\hline Yes & 97 & $97(100)$ & & $97(100)$ & \\
\hline No & 76 & $62(81.6)$ & 0.000 & $54(71.1)$ & 0.000 \\
\hline
\end{tabular}

moderaterly- and poorly-differentiated cases were 51, 41 and 75 , respectively. Initial evalutions showed that 70 cases $(40.5 \%)$ with ESCC had stages I and II disease, whereas 103 cases $(59.5 \%)$ had stages III and IV disease. Finally, 97 cases $(56.1 \%)$ with lymph node metastasis and 76 cases $(43.9 \%)$ without lymph node metastasis were utilized in the study.

The expression of Tiam1 protein in ESCC tissues. Tiam1 protein expression was investigated in 173 cases of primary ESCC samples, 95 cases of para-carcinoma samples and 173 cases of paired normal esophageal samples by immunohistochemistry method. In ESCC tissues, 91.91\% (159 of 173) of samples exhibited positive Tiam1 staining, whereas $8.09 \%$ (14 of 173) of ESCC tissues were not immunoreactive for Tiam 1 . In contrast, only $49.47 \%$ (47 of 95) of paracarcinoma samples and $9.25 \%$ (16 of 173) of normal esophageal tissues displayed positive Tiam1 staining. Compared to those of para-carcinoma and normal esophageal tissues, there was obvious increase in Tiam 1 protein expression in ESCC tissues $(\mathrm{P}<0.05)$ (Fig. 1), indicating that Tiam 1 expression at a high level in ESCC tissues may be closely associated with the occurrence and development of ESCC.

The expression of Tiam1 mRNA in ESCC tissues. In situ hybridization method was used to measure the Tiam1 mRNA expression in 173 cases of primary ESCC samples, 95 cases of para-carcinoma samples and 173 cases of paired normal esophageal samples. The results revealed that positive ratios of Tiam 1 mRNA expression were $87.28 \%$ (151 of 173) in primary ESCC tissues, $41.05 \%$ (39 of 95) in para-carcinoma tissues and $6.94 \%$ (12 of 173) in normal esophageal tissues
(Fig. 2), respectively, suggesting overexpression of Tiam1 mRNA may play a central role in the development of ESCC.

The association between Tiaml expression and clinicopathologic characteristics. To further identify the potential role of Tiam1 in the occurrence and development of ESCC, we analyzed the correlations between Tiam 1 mRNA and protein expressions in ESCC and clinicopathologic parameters including age, gender, histologic grade, clinical staging and lymph node metastasis. We found that Tiam 1 mRNA and protein expressions were both closely associated with histologic grade, clinical stage and lymph node metastasis (all $\mathrm{P}<0.05$ ), but not age and gender (both $\mathrm{P}>0.05$ ) (Table II), notably, in all poorly-differentiated samples and the patients with lymph node metastases, positive expression ratios of Tiam 1 mRNA and protein were both $100 \%$ (Table II), implying Tiam1 expressions with intense mRNA and protein staining may be tightly associated with malignant degree and invasiveness and metastasis of ESCC.

RT-PCR and Western blotting analysis for Tiam1 expression. Randomly 3 cases of ESCC and paired normal epithelium tissues were selected to investigate the relative levels of Tiam1 mRNA and protein by semi-quantitative RT-PCR and Western blotting methods. The results demonstrated that relative levels of Tiam1 mRNA and protein in ESCC tissues were significantly higher than those in normal esophageal tissues (Figs. 3 and 4), indicating overexpression of Tiam1 gene may play an essential role in the occurrence and development of ESCC. 

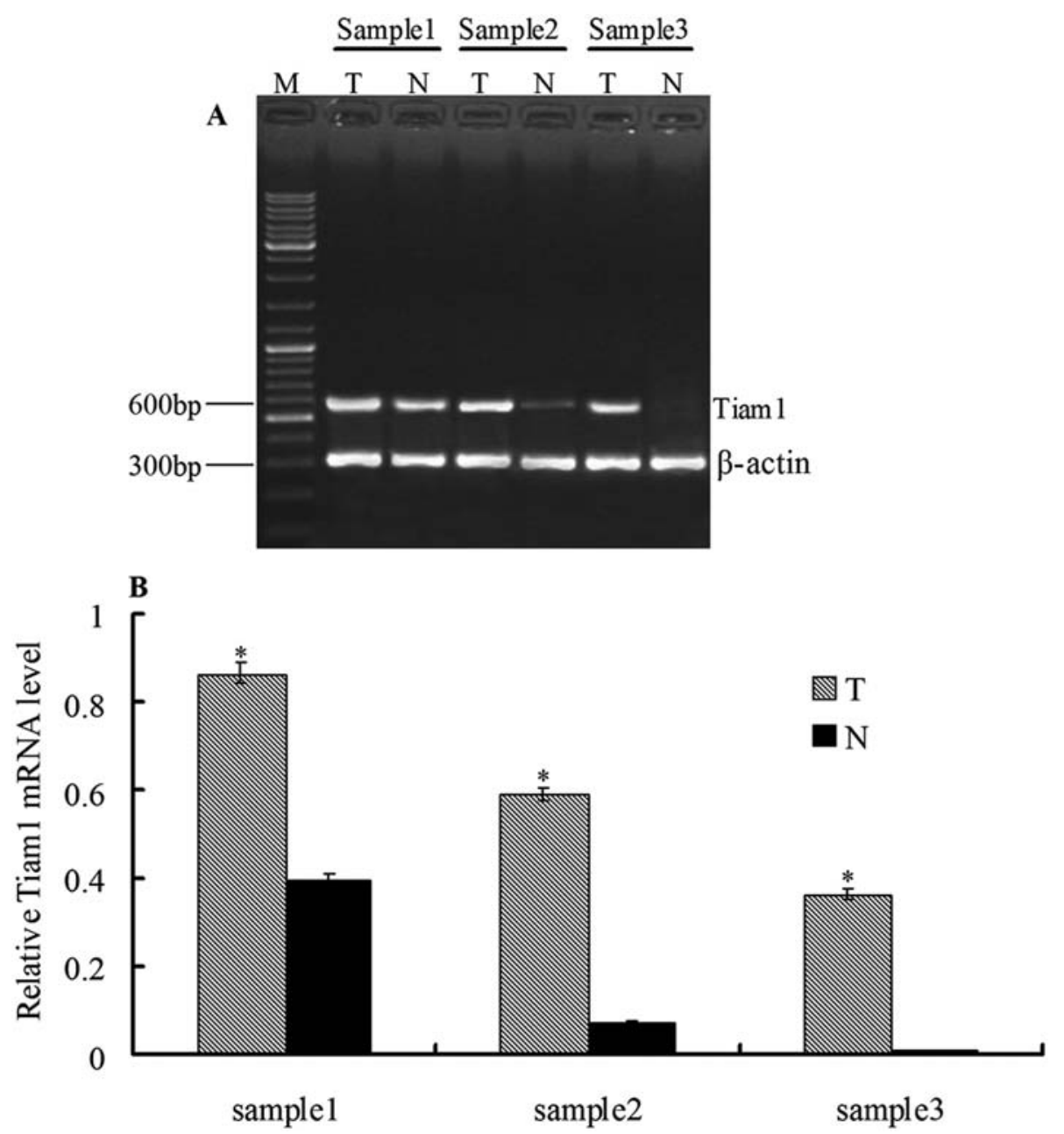

Figure 3. Relative expression analysis of Tiam1 mRNA in three representative cases of ESCC tissues (T) and the adjacent normal esophageal tissues (N). (A) The relative expression of Tiam1 mRNA was analyzed by semi-quantitative RT-PCR. (B) Semi-quantitive values of three independently repeated RT-PCR were statistically analyzed by densitometry using Gene Tools software, are expressed as the means \pm SD. ${ }^{*} \mathrm{P}<0.05$, compared to expression of Tiam1 in normal esophageal tissues.

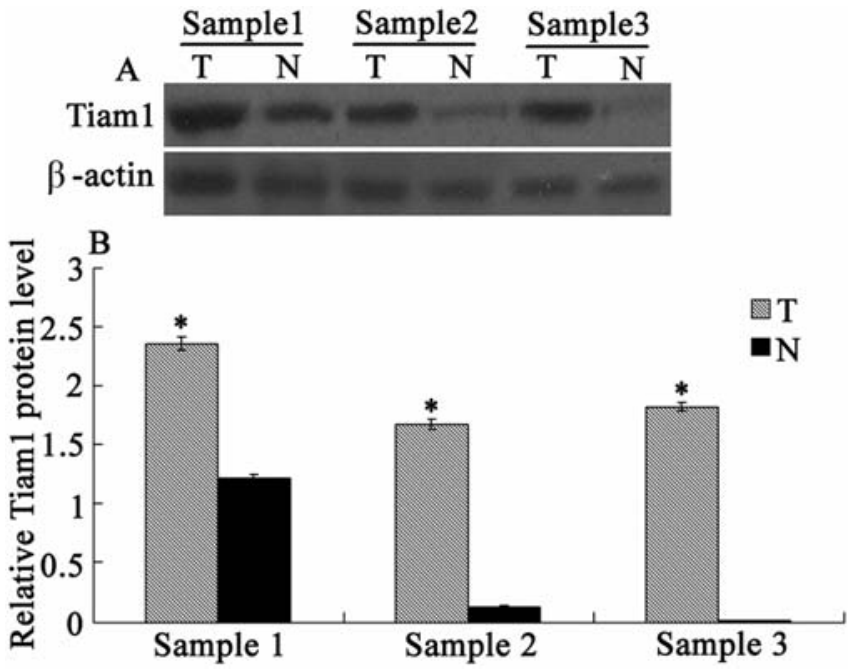

Figure 4. Relative expression analysis of Tiam1 protein in three representative cases of ESCC tissues (T) and the adjacent normal esophageal tissues (N). (A) The relative expression of Tiam1 protein was analyzed by Western blotting. Expression of $\beta$-actin was simultaneously used as an internal control. (B) Semi-quantitive values of three independently repeated Western blotting were statistically analyzed by densitometry using Gene Tools software, are expressed as the means $\pm \mathrm{SD}$. "P $\mathrm{P}<0.05$, compared to expression of Tiam1 in normal esophageal tissues.
Effect of Tiaml expression on prognosis of the patients with ESCC. The relationship between expressions of Tiam1 mRNA and protein and survival time of the patients with ESCC was evaluated by Kaplan-Meier survival curve. The mean follow-up period of all the patients with ESCC in this study was 45 months (range from 1 to 60 months). The results revealed that the patients with high levels of Tiam1 mRNA and protein had a poorer prognosis than those with low levels of Tiam 1 mRNA and proteins, and there was a significant difference between Tiam1 expression and survival time of the patients with ESCC (both $\mathrm{P}<0.01$ ) (Fig. 5), indicating that Tiam1 may be a good prognostic marker in the diagnosis of ESCC.

\section{Disscussion}

Originally, Tiam1, as one of guanine nucleotide exchange factors (GEFs), was identified by retroviral insertional mutagenesis and selected for its invasive cell behavior in vitro $(20,21)$. Tiam1 is involved in a large number of cellular events, such as cytoskeleton reorganization, cell adhesion, cell migration, invasion, metastasis and carcinogenesis, suggesting Tiam1 may play a pivotal role in the occurrence and develop- 
A

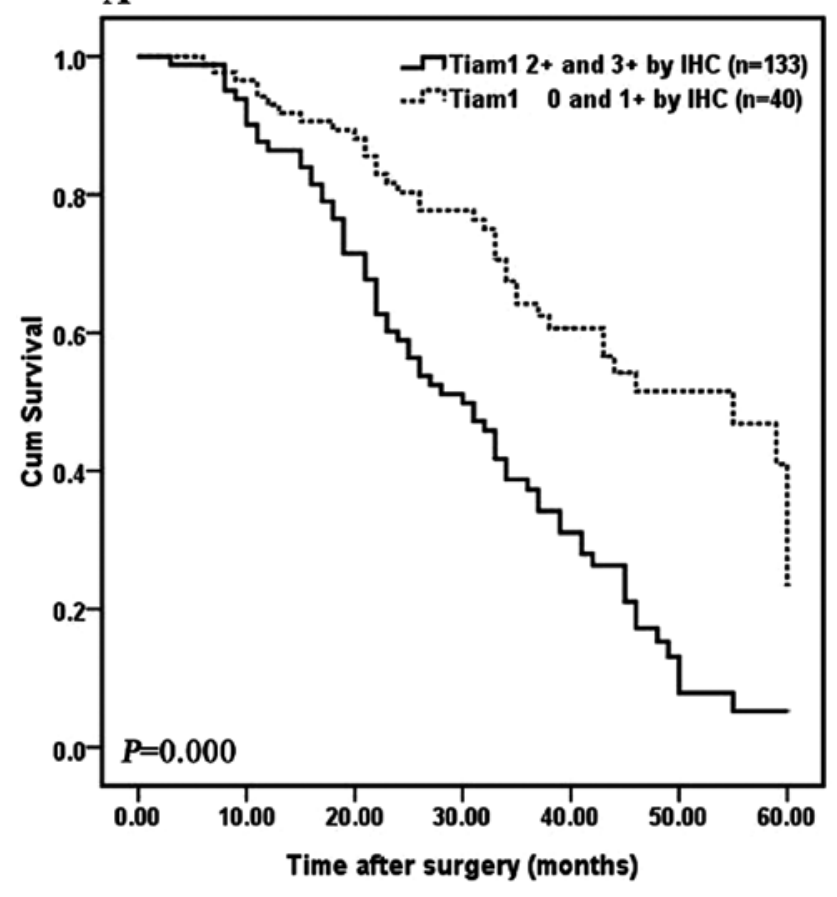

B

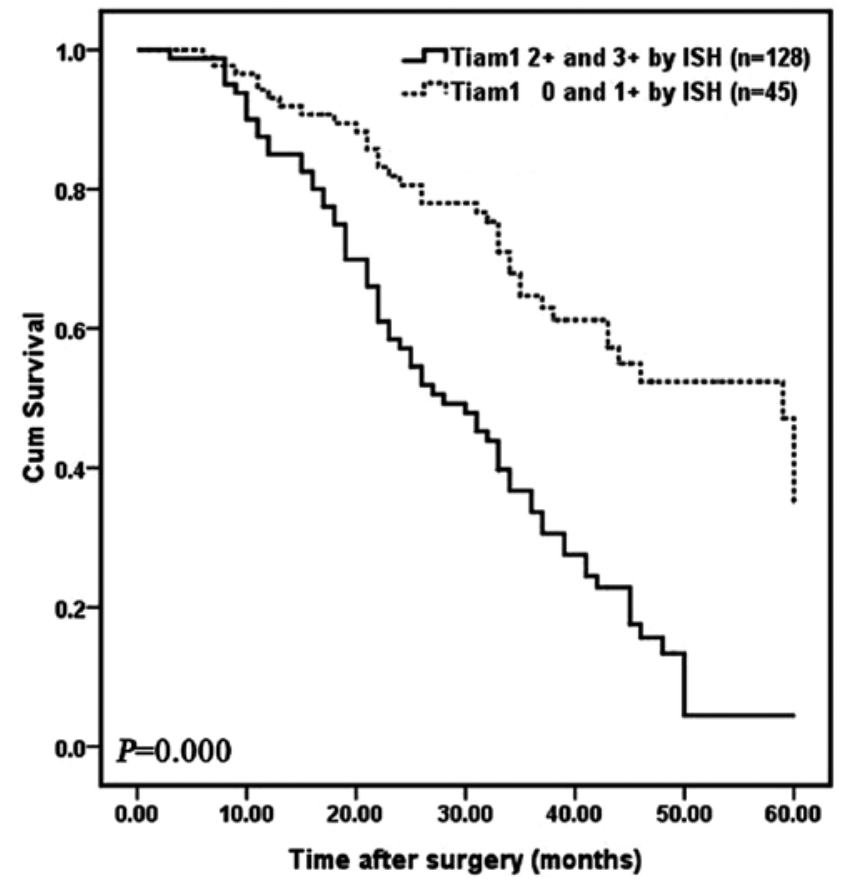

Figure 5. Effect of Tiam1 expression on prognosis of the patients with ESCC by Kaplan-Meier plots. (A) IHC analysis for the association between Tiam1 protein expression and survival time of the patients with ESCC. (B) ISH analysis for the relationship between Tiam1 protein expression and survival time of the patients with ESCC. There was a significant difference between the two curves by log-rank test (Mantel-Cox) $(\mathrm{P}<0.01)$.

ment of tumors. The malignant degree and invasive and metastasis phenotype of ESCC, determined by multiple characteristics such as tumor cell motility and membrane perturbations, is easily linked to changes of specific molecules Tiam1 related to invasiveness and metastasis, however, up to now, the role of Tiam1 in ESCC has not yet investigated worldwide. To our knowledge, this is the first comprehensive description of Tiam1 expression in ESCC tissues by immunohistochemistry, in situ hybridization, semi-quantitative RT-PCR and Western blotting methods, and analysis of the association between Tiam1 expression and the prognosis of the patients with ESCC.

It is well documented that Tiam1, one important regulator of Rho GTPase functions in tumor cells (22), induces T-lymphoma invasion and metastasis, but also inhibits migration and invasion of human renal cell carcinoma through Rac activation $(20,23,24)$. Besides, overexpression of Tiam1 in metastatic melanoma cells converts the constitutive mesenchymal phenotype into an epithelial-like phenotype (25), and, most importantly, is considered as the useful indicator for predicting the prognosis of hepatocellular carcinoma (15), implying the crucial role of Tiam1 in melanoma and hepatocellular carcinoma. Adithi et al reported significantly increased Tiam1 expression in invasive retinoblastoma (14). Malliri et al reported a cross-talk between Tiam1/Rac and the canonical Wnt-signaling pathway that affects intestinal tumor formation and progression (26), indicating Tiam1 play an important role in tumor signaling pathway. Whether these functions of Tiam 1 can be extended to ESCC remains elusive, in the current study, positive Tiam1 protein staining was found in $91.91 \%$ (159 of 173) of the analyzed ESCC cases, whereas only $49.47 \%$ (47 of 95) of paracarcinoma tissues and $9.25 \%$ (16 of 173) of normal esophageal tissues displayed positive Tiam1 protein expression. Additionally, as for Tiam 1 mRNA expression, the result of ISH revealed that positive ratios of Tiam 1 mRNA expression were $87.28 \%$ (151 of 173) in ESCC tissues, 41.05\% (39 of 95) in paracarcinoma tissues and $6.94 \%$ (12 of 173) in normal esophageal tissues, respectively. Compared to those of paracarcinoma and normal esophageal tissues, there was obvious increase in Tiam1 protein and mRNA expressions in ESCC tissues $(\mathrm{P}<0.05)$, indicating that Tiam1 expression at a high level in ESCC tissues may be closely associated with the occurrence and development of ESCC. Moreover, randomly selected 3 cases of ESCC and normal esophageal tissues were used to further investigate the Tiam1 level by semiquantitative RT-PCR and Western blotting methods, the result demonstrated that the levels of Tiam 1 mRNA and protein in ESCC tissues were significantly higher than those in normal esophageal tissues. Notably, expressions of Tiam1 mRNA and protein were presented with intense staining in all poorly-differentiated and the patients with lymph node metastases samples investigated, indicating overexpression of Tiam1 may be tightly associated with malignant degree and invasiveness and metastasis of ESCC.

Increasing evidence has demonstrated that Tiam 1 is tightly related to lymph node metastasis, histological differentiation, clinical stage and disease recurrence. Yang et al recently reported that overexpression of Tiam1 was significantly correlated with vascular invasion status $(\mathrm{P}=0.02)$, 
intrahepatic metastasis status $(\mathrm{P}=0.009)$ and histological differentiation $(\mathrm{P}=0.008)$ of patients with hepatocellular carcinoma (15). In addition, Tiam1 was overexpressed in nasopharyngeal carcinoma cells and their protein up-regulation was significantly associated with stage and grading $(\mathrm{P}<0.05$ for all) (12). Strong Tiam1 overexpression in prostate carcinomas relative to the respective benign prostatic epithelium was statistically significantly associated with disease recurrence $(\mathrm{P}=0.016)$, the presence of lymph vessel invasion $(\mathrm{P}=0.031)$ and high Gleason scores (GS) (27). Moreover, Tiam1 expression was found to correlate with a high tumor grade in a small number of breast cancer tissue samples (28). To further elucidate the potential role of Tiam1 in the occurrence and development of ESCC, we analyzed the relationship between expressions of Tiam 1 mRNA and protein and clinicopathologic parameters including age, gender, histologic grade, clinical staging and lymph node metastasis. We found that Tiam 1 protein and mRNA expressions were both closely associated with histologic grade (both $\mathrm{P}=0.000$ ), clinical stage $(\mathrm{P}=0.014$ and 0.000 , respectively) and lymph node metastasis (both $\mathrm{P}=0.000)$, but not gender $(\mathrm{P}=0.462$ and 0.181$)$ and age $(\mathrm{P}=0.779$ and 0.755 , respectively), suggesting Tiam 1 may play an important role in the occurrence and development of ESCC, however, precise molecular mechanisms have yet remained to be elucidated.

Although numerous molecular markers including p53 (29), Ki-67 (30) and cyclin D1 (31) have been utilized to evaluate survival probabilities of patients with cancer, few transfer to widespread clinical use. Yang et al found that hepatocellular carcinoma patients with strong Tiam1 expression had shorter disease-specific survival than those with weak expression $(\mathrm{P}=0.01)$. Multivariate analysis also showed that Tiam1 overexpression may be a predictor of poor prognosis $(\mathrm{P}=0.02)(15)$. Up-regulation of Tiam1 protein was associated with lower disease-free and overall survival rates $(\mathrm{P}<0.001)$; implying up-regulation of Tiam1 protein may play a critical role in tumor progression of nasopharyngeal carcinoma, and work as a prognostic factor for the patients with nasopharyngeal carcinoma (12). Certainly, prognostic value of Tiam1 has extended to a great number of different tumors including prostate carcinoma (27) and gastric carcinoma (32), but not ESCC. In this study, the patients of ESCC with high levels of Tiam1 mRNA and protein had a poorer prognosis than those with low levels of Tiam 1 mRNA and proteins, and there was a significant difference between Tiam1 expression and survival time of the patients with ESCC (both $\mathrm{P}<0.01$ ), suggesting Tiam1 mRNA and protein at high level may be an independent prognostic marker for poorer survival in all investigated patients with ESCC.

In summary, our study suggests high Tiam1 expression to be an independent prognostic marker for poorer survival in the patients with ESCC. High level Tiam1 was closely associated with maglinant phenotype and lymph node metastasis of ESCC. Further work is needed to elucidate the important biological significance of high Tiam1 expression and better outcome, which will make Tiam1 as an extremely attractive molecular therapeutic target for ESCC.

\section{References}

1. Kamangar F, Dores GM and Anderson WF: Patterns of cancer incidence, mortality, and prevalence across five continents: defining priorities to reduce cancer disparities in different geographic regions of the world. J Clin Oncol 24: 2137-2150, 2006.

2. Parkin DM, Bray FI and Devesa SS: Cancer burden in the year 2000. The global picture. Eur J Cancer 37 (Suppl. 8): S4-S66, 2001.

3. Parkin DM, Laara E and Muir CS: Estimates of the worldwide frequency of sixteen major cancers in 1980. Int J Cancer 41: 184-197, 1988

4. Yang CS: Research on esophageal cancer in China: a review. Cancer Res 40: 2633-2644, 1980.

5. Layke JC and Lopez PP: Esophageal cancer: a review and update. Am Fam Physician 73: 2187-2194, 2006.

6. Parkin DM, Bray F, Ferlay J and Pisani P: Global cancer statistics, 2002. CA Cancer J Clin 55: 74-108, 2005.

7. Enzinger PC and Mayer RJ: Esophageal cancer. N Engl J Med 349: 2241-2252, 2003

8. Michiels F, Habets GG, Stam JC, van der Kammen RA and Collard JG: A role for Rac in Tiam1-induced membrane ruffling and invasion. Nature 375: 338-340, 1995.

9. Hordijk PL, ten Klooster JP, van der Kammen RA, Michiels F, Oomen LC and Collard JG: Inhibition of invasion of epithelial cells by Tiam1-Rac signaling. Science 278: 1464-1466, 1997.

10. Sander EE, van Delft S, ten Klooster JP, Reid T, van der Kammen RA, Michiels F and Collard JG: Matrix-dependent Tiam1/Rac signaling in epithelial cells promotes either cell-cell adhesion or cell migration and is regulated by phosphatidylinositol 3-kinase. J Cell Biol 143: 1385-1398, 1998.

11. Sander EE, ten Klooster JP, van Delft S, van der Kammen RA and Collard JG: Rac downregulates Rho activity: reciprocal balance between both GTPases determines cellular morphology and migratory behavior. J Cell Biol 147: 1009-1022, 1999.

12. Qi Y, Huang B, Yu L, Wang Q, Lan G and Zhang Q: Prognostic value of Tiam 1 and Rac1 overexpression in nasopharyngeal carcinoma. ORL J Otorhinolaryngol Relat Spec 71: 163-171, 2009.

13. Minard ME, Kim LS, Price JE and Gallick GE: The role of the guanine nucleotide exchange factor Tiam1 in cellular migration, invasion, adhesion and tumor progression. Breast Cancer Res Treat 84: 21-32, 2004

14. Adithi M, Venkatesan N, Kandalam M, Biswas J and Krishnakumar S: Expressions of Rac1, Tiam1 and Cdc42 in retinoblastoma. Exp Eye Res 83: 1446-1452, 2006.

15. Yang W, Lv S, Liu X, Liu H and Hu F: Up-regulation of Tiam1 and Rac1 correlates with poor prognosis in hepatocellular carcinoma. Jpn J Clin Oncol 40: 1053-1059, 2010.

16. Liu HT, Wang N, Wang X and Li SL: Overexpression of Pim-1 is associated with poor prognosis in patients with esophageal squamous cell carcinoma. J Surg Oncol 102: 683-688, 2010.

17. Zhou C, Liu S, Zhou X, Xue L, Quan L, Lu N, Zhang G, Bai J, Wang Y, Liu Z, Zhan Q, Zhu H and Xu N: Overexpression of human pituitary tumor transforming gene (hPTTG), is regulated by beta-catenin /TCF pathway in human esophageal squamous cell carcinoma. Int J Cancer 113: 891-898, 2005.

18. Zeng H, Xu L, Xiao D, Zhang H, Wu X, Zheng R, Li Q, Niu Y, Shen $\mathrm{Z}$ and Li E: Altered expression of ezrin in esophageal squamous cell carcinoma. J Histochem Cytochem 54: 889-896, 2006.

19. Li S, Xi Y, Zhang H, Wang Y, Wang X, Liu H and Chen K: A pivotal role for Pim-1 kinase in esophageal squamous cell carcinoma involving cell apoptosis induced by reducing Akt phosphorylation. Oncol Rep 24: 997-1004, 2010.

20. Habets GG, Scholtes EH, Zuydgeest D, van der Kammen RA, Stam JC, Berns A and Collard JG: Identification of an invasioninducing gene, Tiam-1, that encodes a protein with homology to GDP-GTP exchangers for Rho-like proteins. Cell 77: 537-549, 1994.

21. Habets GG, van der Kammen RA, Stam JC, Michiels F and Collard JG: Sequence of the human invasion-inducing TIAM1 gene, its conservation in evolution and its expression in tumor cell lines of different tissue origin. Oncogene 10: 1371-1376, 1995.

22. Mertens AE, Roovers RC and Collard JG: Regulation of Tiam1Rac signalling. FEBS Lett 546: 11-16, 2003.

23. Michiels F and Collard JG: Rho-like GTPases: their role in cell adhesion and invasion. Biochem Soc Symp 65: 125-146, 1999. 
24. Engers R, Springer E, Michiels F, Collard JG and Gabbert HE: Rac affects invasion of human renal cell carcinomas by upregulating tissue inhibitor of metalloproteinases (TIMP)- 1 and TIMP-2 expression. J Biol Chem 276: 41889-41897, 2001.

25. Uhlenbrock K, Eberth A, Herbrand U, Daryab N, Stege P, Meier F, Friedl P, Collard JG and Ahmadian MR: The RacGEF Tiam1 inhibits migration and invasion of metastatic melanoma via a novel adhesive mechanism. J Cell Sci 117: 4863-4871, 2004.

26. Malliri A, Rygiel TP, van der Kammen RA, Song JY, Engers R, Hurlstone AF, Clevers $\mathrm{H}$ and Collard JG: The rac activator Tiam1 is a Wnt-responsive gene that modifies intestinal tumor development. J Biol Chem 281: 543-548, 2006.

27. Engers R, Mueller M, Walter A, Collard JG, Willers R and Gabbert HE: Prognostic relevance of Tiam1 protein expression in prostate carcinomas. Br J Cancer 95: 1081-1086, 2006.

28. Adam L, Vadlamudi RK, McCrea P and Kumar R: Tiam1 overexpression potentiates heregulin-induced lymphoid enhancer factor-1/beta-catenin nuclear signaling in breast cancer cells by modulating the intercellular stability. J Biol Chem 276: 28443-28450, 2001.
29. Wang DY, Xiang YY, Tanaka M, Li XR, Li JL, Shen Q, Sugimura $\mathrm{H}$ and Kino I: High prevalence of p53 protein overexpression in patients with esophageal cancer in Linxian, China and its relationship to progression and prognosis. Cancer 74 : 3089-3096, 1994.

30. Ikeda G, Isaji S, Chandra B, Watanabe $M$ and Kawarada $Y$ : Prognostic significance of biologic factors in squamous cell carcinoma of the esophagus. Cancer 86: 1396-1405, 1999.

31. Yoshida K, Kawami H, Yamaguchi Y, Kuniyasu H, Nishiyama M, Hirai T, Yanagihara K, Tahara E and Toge T: Retrovirally transmitted gene therapy for gastric carcinoma using herpes simplex virus thymidine kinase gene. Cancer 75 : 1467-1471, 1995.

32. Walch A, Seidl S, Hermannstadter C, Rauser S, Deplazes J, Langer R, von Weyhern CH, Sarbia M, Busch R, Feith M, Gillen S, Hofler H and Luber B: Combined analysis of Rac1, IQGAP1, Tiam1 and E-cadherin expression in gastric cancer. Mod Pathol 21: 544-552, 2008. 